\title{
Cavitation-induced ignition of cryogenic hydrogen-oxygen fluids
}

\author{
V. V. Osipov ${ }^{1,2}$, C. B. Muratov ${ }^{3}$, E. Ponizovskya-Devine ${ }^{1,2}$, M.Foygel $^{4}$, V. N. Smelyanskiy ${ }^{1}$ \\ ${ }^{1}$ Intelligent Systems Division, DESSH Branch, NASA Ames Research Center, MS 269-1, Moffett Field, CA 94035 \\ ${ }^{2}$ Mission Critical Technologies, Inc., 2041 Rosecrans Avenue, Suite 225, El Segundo, CA 90245 and \\ ${ }^{3}$ Department of Mathematical Sciences, New Jersey Institute of Technology, Newark, NJ 07102 \\ ${ }^{4}$ South Dakota School of Mines and Technology, Rapid City, SD, 57701
}

(Dated: May 25, 2022)

\begin{abstract}
The Challenger disaster and purposeful experiments with liquid hydrogen (H2) and oxygen (Ox) tanks demonstrated that cryogenic $\mathrm{H} 2 / \mathrm{Ox}$ fluids always self-ignite in the process of their mixing. Here we propose a cavitation-induced self-ignition mechanism that may be realized under these conditions. In one possible scenario, self-ignition is caused by the strong shock waves generated by the collapse of pure Ox vapor bubble near the surface of the Ox liquid that may initiate detonation of the gaseous $\mathrm{H} 2 / \mathrm{Ox}$ mixture adjacent to the gas-liquid interface. This effect is further enhanced by $\mathrm{H} 2 / \mathrm{Ox}$ combustion inside the collapsing bubble in the presence of admixed $\mathrm{H} 2$ gas.
\end{abstract}

The source for the formation of flames in the cryogenic hydrogen/oxygen $(\mathrm{H} 2 / \mathrm{Ox})$ fuel mixture during the Challenger disaster in 1986 still remains a mystery. The fireball which caused the orbiter's destruction appeared near the ruptured intertank section between the liquid H2 (LH2) and liquid Ox (LOx) tanks, but not near the hot jets from the nozzles 12 . Purposeful experiments with LOx and LH2 tanks carried out by $\mathrm{NASA}^{3}$ showed that cryogenic $\mathrm{H} 2 / \mathrm{Ox}$ mixtures always self-ignite when the flows of cryogenic fluids containing gaseous hydrogen (GH2), gaseous oxygen (GOx) and LH2 mix with a turbulent LOx stream. Since this effect can lead to catastrophic events, understanding its mechanisms is a problem of great importance.

In this Letter we propose a cavitation-induced selfignition mechanism of cryogenic H2/Ox fluids. Cavitation is the formation and compression of vapor bubbles in flowing liquids driven by abrupt pressure variations. Due to inertial motion of the liquid this process leads to a rapid collapse of the bubbles and spiking of the gas temperature and pressure inside the bubbles, producing strong shock waves ${ }^{45}$. Here we discuss possible scenarios of cavitation-induced ignition in cryogenic $\mathrm{Ox} / \mathrm{H} 2$ fluids. We concentrate on the most transparent scenario related to the collapse of a vapor bubble in the $\mathrm{Ox}$ liquid near the interface between LOx and the GH2/GOx mixture.

Vapor bubbles, most likely with admixed GH2, can be created in the falling LOx blobs as a result of mixing of gaseous $\mathrm{H} 2$ and $\mathrm{Ox}$ with a turbulent stream of liquid Ox. A pressure jump between LOx and the bubbles may arise, for example, due to shock waves arising as a result of an impact of a LOx blob against a solid object (Fig, 1 $)$. The overpressure in such a shock wave is of order $\Delta p \simeq \frac{1}{2} \rho_{L} v^{2} \gtrsim 2 \mathrm{~atm}$ even for moderate velocities $v \gtrsim 20 \mathrm{~m} / \mathrm{s}$ of the liquid. Such a "weak" shock wave cannot induce ignition of the GH2/GOx mixture directly, but it can initiate cavitation collapse of the vapor bubbles inside LOx. The below computations show that such weak initiating shock waves can lead to the formation of bubbles of a small radius $R_{\min } \simeq 0.1 \mathrm{~mm}$ with huge pressures $p \gtrsim 1000 \mathrm{~atm}$ and temperatures $T \gtrsim 2500 \mathrm{~K}$ inside (Fig. 2). This causes ignition of the GOx/GH2 mixture inside the bubble. A strong secondary shock wave generated by the cavitation bubble collapse near the LOx interface may then propagate into the gaseous $\mathrm{H} 2 / \mathrm{Ox}$ mixture next to the LOx interface (Fig. 10). We demonstrate that such a localized shock wave is sufficient to induce detonation in cryogenic GH2/GOx mixtures (Fig. 3).

To simulate the bubble collapse, we used the standard fluid dynamics equations describing conservation of the mass, energy and momentum under the assumption of spherical symmetry $\stackrel{46}{ }$. We treated the liquid as incompressible and inviscid, neglected surface tension at the interface, treated the gas phase as a mixture of ideal gases and took into account diffusion and thermodiffusion of the admixed GH2 (see supplementary material for more detail). We also modeled combustion inside the bubble, using a simplified model based on the assumption that the burning rate is limited by the initiation reactions $\mathrm{H}_{2}+\mathrm{O}_{2} \rightarrow \mathrm{OH}+\mathrm{OH}$ and $\mathrm{H}_{2}+\mathrm{O}_{2} \rightarrow \mathrm{HO}_{2}+\mathrm{H}$, which have the lowest rates. Thus, we modeled the $\mathrm{GH} 2 / \mathrm{GOx}$ combustion by a brutto reaction $\mathrm{H}_{2}+\mathrm{O}_{2} \rightarrow \mathrm{H}_{2} \mathrm{O}+\frac{1}{2} \mathrm{O}_{2}$ with the rate $G_{\text {comb }}(T)=c_{H 2} c_{O x}\left[1.1 \cdot 10^{8} \exp (-19680 / T)+\right.$ 1.48. $\left.T^{2.433} \exp (-26926 / T)\right] \mathrm{m}^{3} /(\mathrm{mol} \cdot \mathrm{sec})$, where $c_{H 2}$ and $c_{O x}$ are the molar concentrations of GH2 and GOx, respectively, and $T$ is in degrees Kelvin ${ }^{7}$. We note that this approximation is close to the one-step mechanism of Mitani and Williams ${ }^{8}$. Also, the model predicts the same steady detonation wave parameters as those obtained ${ }^{3}$ with the help of the model taking into account 17 main chain reactions of GOx/GH2 mixture combustion ${ }^{9}$.

Our simulations show that under the action of initiating shock wave with overpressure $\Delta p \geq 0.15 \mathrm{~atm}$ the maximum pressure and temperature in pure vapor bubbles of initial radius $R_{0} \gtrsim 2 \mathrm{~mm}$ collapsing in LOx exceed $1500 \mathrm{~atm}$ and $800 \mathrm{~K}$, respectively, when the bubble radius reaches its minimum value of $R_{\min } \simeq 0.1 \mathrm{~mm}$ (curve 1 in Fig.2). With the increase of $\Delta p$ the values of $p_{\max }$ and $T_{\max }$ increase and the value of $R_{\min }$ decreases $\left(R_{\min } \leq 0.05 \mathrm{~mm}\right.$ at $\left.\Delta p \geq 0.5 \mathrm{~atm}\right)$. The lower the value of $R_{\min }$, the harder it is to achieve ignition. The presence in the bubble of even a small amount of noncondensable GH2 sharply decreases the values of $p_{\max }$ 
and $T_{\max }$. However, at large enough $\Delta p \geq 0.5 \mathrm{~atm}$ and $R_{0} \gtrsim 2 \mathrm{~mm}$ the bubble collapse leads to high temperatures and ignition of the $\mathrm{Ox} / \mathrm{H} 2$ mixture in the bubble. As a result of this local explosion the values of $p_{\max }$ and $T_{\max }$ in the bubble reach gigantic values $p_{\max } \geq 8000$ atm and $T_{\max } \geq 3500 \mathrm{~K}$ (Fig. 2 ). We note that in reality the super-hot and super-compressed $\mathrm{O}, \mathrm{H}, \mathrm{OH}$ species forming in the process of $\mathrm{GH} 2 / \mathrm{GOx}$ combustion 9 inside the bubble may be ejected into the space above the LOx surface and easily ignite the $\mathrm{GH} 2 / \mathrm{GOx}$ mixture nearby.

The same equations of gas dynamics used in the cavitation simulations were also employed to analyze the ignition of GH2/GOx mixtures by a localized strong shock wave generated by the collapsing bubble. We envision a hemispherical shock wave propagating in the unconfined GH2/GOx mixture above the LOx-gas interface (see Fig. 1b), initiated by a local increase in gas pressure and temperature within the radius $R_{0} \sim R_{\text {min }}$. We see that the
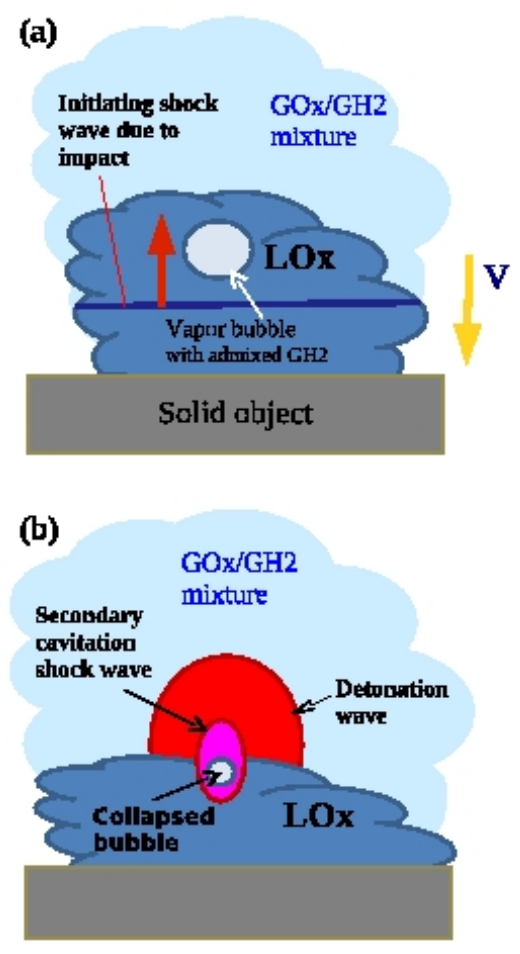

FIG. 1: A scenario of cavitation-induced ignition of GOx/GH2 mixture: (a) an initiating "weak" shock wave in a LOx blob (with a bubble) forms due to its impact with a solid object; (b) collapse of the bubble near the liquid-gas interface under the action of the initiating shock wave and generation of a strong secondary shock wave inducing detonation of the GH2/GOx mixture.
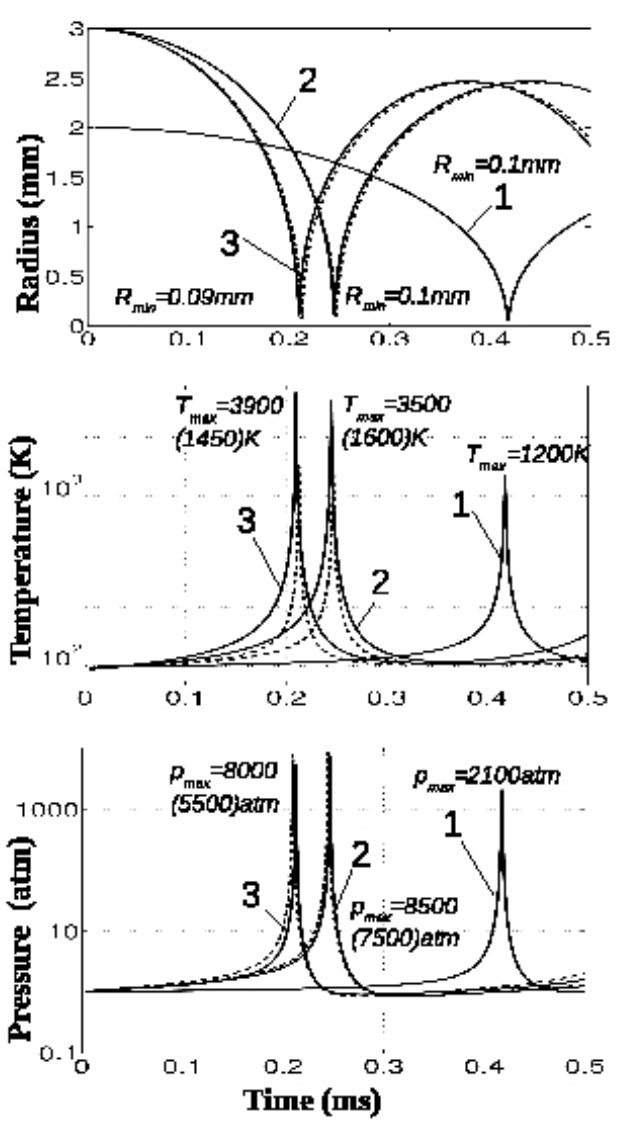

FIG. 2: Bubble collapse dynamics for the initial total gas pressure $p_{0}=1 \mathrm{~atm}$. Other initial parameters are: radius $R_{0}=2$ $\mathrm{mm}$, overpressure of the initiating shock wave $\Delta p=0.25$ atm, GH2 partial pressure $p_{H 2}=0$ (curve 1 ); $R_{0}=3 \mathrm{~mm}$, $\Delta p=1.5 \mathrm{~atm}, p_{H 2}=0.01 \mathrm{~atm}($ curve 2$) ; R_{0}=3 \mathrm{~mm}$, $\Delta p=2.0 \mathrm{~atm}, p_{H 2}=0.015 \mathrm{~atm}$ (curve 3). The dashed lines and the values in parentheses are obtained without considering combustion in the bubble.

local jump of the pressure $p \gtrsim 200$ atm and temperature $T \gtrsim 500 \mathrm{~K}$ in the $\mathrm{GH} 2 / \mathrm{GOx}$ mixture area of radius $R_{0} \gtrsim 0.1 \mathrm{~mm}$ is sufficient to induce detonation in stoichiometric GH2/GOx mixtures (Fig. 3).

We now list other possible cavitation-induced scenarios that may lead to self-ignition of cryogenic H2/Ox fluids upon mixing:

(i) Formation of rarefied-vapor bubbles in LOx.

(ii) Formation of GOx bubbles in LOx with a thin chilled layer near the bubble surface.

(iii) Injection of cold LH2 droplets into "hot" LOx.

(iv) Injection of "hot" LOx droplets into cold LH2.

In scenario (i), rarefied-vapor bubbles form in the $\mathrm{LOx}$ stream impinging on an obstacle and collapse under the 

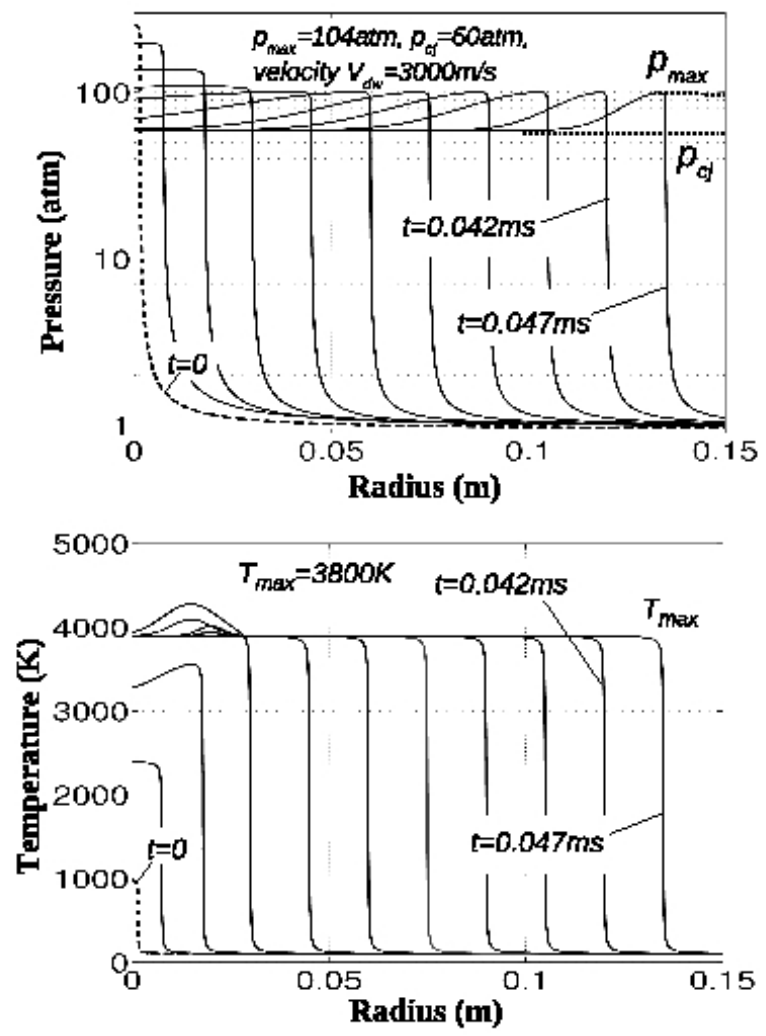

FIG. 3: Formation of cavitation-induced hemispheric detonation wave in stoichiometric gaseous $\mathrm{H} 2 / \mathrm{Ox}$ mixture with temperature $T=100 \mathrm{~K}$ and pressure $p=1 \mathrm{~atm}$. The initial conditions are: temperature $T=1000 \mathrm{~K}$ and pressure $p=250$ atm in the central area with radius $r \leq 0.15 \mathrm{~mm}$ (dashed curves). $p_{c j}$ - steady-state pressure in the detonation wave (Chapman-Jouguet pressure ${ }^{(9}$ )

liquid pressure. In scenario (ii) bubbles may form as a result of an impact of two large LOx blobs whose surfaces were chilled by contact with very cold $(T \simeq 20 \mathrm{~K})$ surrounding GH2. Due to the low near-surface temperature the pressure in the bubble may quickly drop because of intense vapor condensation, leading to a rapid bubble collapse. In scenario (iii) the pressure inside the bubble will quickly grow and may become much greater than the pressure $p_{L}$ in the liquid bulk. As a result, the bubble radius will increase and, due to the inertial motion of the liquid, the pressure in the bubble can become much less than $p_{L}$. As a consequence, the bubble will start to collapse, and the gas temperature and pressure inside the bubble may achieve very high values, initiating a local explosion and a strong shock wave. Finally, in scenario (iv) heavy droplets of LOx may penetrate deeply into LH2 (a light fluid), causing intense evaporation of LH2 and formation of a GH2/GOx bubble inside LH2 that will grow in size and then collapse due to inertial motion of the liquid. Since the critical temperature $T_{c}=33.2 \mathrm{~K}$ of $\mathrm{H} 2$ is significantly below the freezing temperature $T_{m} \simeq 54 \mathrm{~K}$ of LOx, the evaporation of LH2 in contact with LOx may acquire an explosive character, resulting in even more dramatic outcomes.

To summarize, we have identified a possible mechanism of ignition in cryogenic $\mathrm{H} 2 / \mathrm{Ox}$ fluids which relies on the generation of strong shock waves by the cavitational collapse of vapor bubbles close to the liquid-gas interface in the process of cryogenic $\mathrm{H} 2 / \mathrm{Ox}$ mixing. We showed that the presence of LOx blobs surrounded by GH2/GOx mixture may be sufficient to initiate $\mathrm{H} 2 / \mathrm{Ox}$ ignition, including strong detonation waves. We further proposed several other scenarios that include mixing of LH2 with LOx and resulting in even more dramatic consequences. More detailed studies of these mechanisms are currently underway. Finally, we note that the proposed self-ignition mechanisms should be very important for understanding conditions and risks of explosion in cryogenic H2/Ox-based liquid rockets and other space vehicles.

The work of CBM was supported by NASA via grant NNX10AC65G.

\section{APPENDIX: GOVERNING EQUATIONS}

Neglecting the surface tension and treating the liquid as incompressible and inviscid, the equations for the liquid phase may be reduced (see e.g. ${ }^{4}$ ) to a single ordinary differential equation for the bubble radius $R$ :

$$
\begin{aligned}
& R \frac{d^{2} R}{d t^{2}}+\frac{3}{2}\left(\frac{d R}{d t}\right)^{2}+\frac{j}{\rho_{L}} \frac{d R}{d t}+\frac{R}{\rho_{L}} \frac{d j}{d t} \\
= & \frac{p_{m}-p_{L 0}}{\rho_{L}}+\frac{\left(2 \rho_{L}-\rho_{v}\right) j^{2}}{2 \rho_{v} \rho_{L}^{2}},
\end{aligned}
$$

and the advection-diffusion equation for the liquid temperature $T_{L}$ :

$$
\begin{aligned}
& \frac{\partial T_{L}}{\partial t}+\left(\frac{R}{r}\right)^{2}\left(\frac{d R}{d t}+\frac{j}{\rho_{L}}\right) \frac{\partial T_{L}}{\partial r} \\
= & \frac{\kappa_{L}}{c_{L} \rho_{L}} \frac{1}{r^{2}} \frac{\partial}{\partial r}\left(r^{2} \frac{\partial T_{L}}{\partial r}\right) .
\end{aligned}
$$

Here $r \geq R(t)$ is the radial coordinate, $\rho_{L}, c_{L}$ and $\kappa_{L}$ are the liquid density, specific heat and thermal conductivity, respectively, $p_{m}$ and $\rho_{v}$ are the pressure and the vapor mass density, respectively, in the gas mixture at the liquid-gas interface, $p_{L 0}$ is the liquid pressure far from the bubble. The vapor condensation flux $j$ is given by the well-known Hertz-Knudsen equation ${ }^{4}$ :

$$
j=\frac{\alpha\left(p_{v}-p_{s}\left(T_{s}\right)\right)}{\sqrt{2 \pi R_{v} T_{s}}}, \quad p_{s}(T)=p_{c}\left(T_{s} / T_{c}\right)^{\lambda},
$$

where $T_{s}$ and $p_{v}$ are the vapor temperature and pressure, respectively, at the liquid-gas interface, $R_{v}$ is the vapor 
gas constant, $\alpha$ is the accommodation coefficient. Here we used a simple approximation for the dependence of the saturated vapor pressure $p_{s}\left(T_{s}\right)$ on the liquid-gas interface temperature $T_{s}$, where $p_{c}$ and $T_{c}$ are critical pressure and temperature, respectively, of the vapor, and $\lambda$ is a dimensionless parameter ( $\left.\sec ^{10}\right)$. Note that $p_{s}$ depends strongly on $T_{s}$, which significantly affects the bubble dynamics 511 .

In the gas phase, we have the conservation of momentum and energy (again neglecting viscosity effects):

$$
\begin{gathered}
\frac{\partial u_{m}}{\partial t}+u_{m} \frac{\partial u_{m}}{\partial r}=-\frac{1}{\rho_{m}} \frac{\partial p_{m}}{\partial r} \\
\frac{\partial E_{m}}{\partial t}+\frac{1}{r^{2}} \frac{\partial}{\partial r}\left(r^{2} u_{m}\left(p_{m}+E_{m}\right)\right) \\
=\frac{1}{r^{2}} \frac{\partial}{\partial r}\left(\varkappa_{m} r^{2} \frac{\partial T_{m}}{\partial r}\right)+Q_{h} G_{c o m b} .
\end{gathered}
$$

Here $\rho_{m}, u_{m}, p_{m}, T_{m}, E_{m}$ are the density, radial velocity, pressure, temperature, and energy density, respectively, of the gas mixture located at $r \leq R(t)$, and $\varkappa_{m}=\left(\frac{T_{m}}{T_{0}}\right)^{1 / 2} \sum_{i} c_{i} \varkappa_{i}^{0} /\left(\sum_{i} c_{i}\right)$ is the thermal conductivity of the gas mixture as a function of $T_{m}$, the molar concentrations $c_{i}=\left\{c_{\mathrm{H} 2}, c_{\mathrm{O} x}, c_{\mathrm{H} 2 \mathrm{O}}\right\}$ of different molecular components and thermal conductivities of pure gas species $\varkappa_{i}^{0}$ at some reference temperature $T_{0}$. Finally, $G_{\text {comb }}$ is the combustion rate and $Q_{h}$ is the combustion heat. Treating all the gas species as diatomic ideal gases for simplicity, we have

$$
p_{m}=R_{g} T_{m} c_{m}, \quad E=\frac{5}{2} R_{g} T_{m} c_{m}+\frac{1}{2} \rho_{m} u_{m}^{2},
$$

where $c_{m}=\sum_{i} c_{i}$ and $\rho_{m}=\sum_{i} c_{i} M_{i}$ are the total molar concentration and mass density, respectively, of the gas mixture, with $R_{g}$ the universal gas constant and $M_{i}$ the molar masses of the gas species. Note that we kept the kinetic energy term in the expression for $E_{m}$ in order to be able to account for possible rapid onset of combustion inside the bubble.

The dynamics of the GH2/GO2 mixture combustion are described by the continuity equations for the molar concentration of the mixture components $c_{i}$ :

$$
\begin{array}{r}
\frac{\partial c_{H 2 O}}{\partial t}+\frac{1}{r^{2}} \frac{\partial}{\partial r}\left(r^{2} c_{H 2 O} u_{m}\right)=G_{c o m b}, \\
\frac{\partial c_{O x}}{\partial t}+\frac{1}{r^{2}} \frac{\partial}{\partial r}\left(r^{2} c_{O x} u_{m}\right)=-\frac{1}{2} G_{c o m b}, \\
\frac{\partial c_{H 2}}{\partial t}+\frac{1}{r^{2}} \frac{\partial}{\partial r}\left(r^{2} c_{H 2} u_{m}\right)+G_{c o m b} \\
=\frac{1}{r^{2}} \frac{\partial}{\partial r}\left\{r^{2} D_{H 2}\left(\frac{\partial c_{H 2}}{\partial r}-\frac{c_{H 2}}{2 T_{m}} \frac{\partial T_{m}}{\partial r}\right)\right\},
\end{array}
$$

where $D_{H 2}=\left(T_{m} / T_{0}\right)^{3 / 2}\left(p_{0} / p_{m}\right) D_{H 2}\left(T_{0}, p_{0}\right) \quad\left(\sec ^{\sqrt{12}}\right)$. Note that in the last equation we included the effect of GH2 diffusion, which may be significant due to high diffusivity of hydrogen and its role as a non-condensable gas during the bubble collapse.

Finally, the boundary and the initial conditions for the equations above are:

$$
\begin{aligned}
\left.\frac{\partial T_{m}}{\partial r}\right|_{r=0} & =0,\left.\quad\left(\kappa_{L} \frac{\partial T_{L}}{\partial r}-\kappa_{m} \frac{\partial T_{m}}{\partial r}\right)\right|_{r=R}=j q_{h}, \\
\left.T_{m}\right|_{t=0} & =\left.T_{L}\right|_{t=0}=T_{L 0},\left.\quad T_{L}\right|_{r=R}=\left.T_{m}\right|_{r=R}=T_{s}, \\
\left.T_{L}\right|_{r \gg R} & =T_{L 0},\left.\quad \frac{\partial c_{i}}{\partial r}\right|_{r=0}=0,\left.\quad c_{i}\right|_{t=0}=c_{i}^{0}, \\
\left.u_{m}\right|_{r=0} & =0,\left.\quad u_{m}\right|_{r=R}=\frac{d R}{d t}-\frac{j}{M_{O x} c_{O x}},\left.\quad u_{m}\right|_{t=0}=0 \\
\left.\frac{j c_{H 2}}{M_{O x} c_{O x}}\right|_{r=R} & =\left.D_{H 2}\left(\frac{\partial c_{H 2}}{\partial r}-\frac{c_{H 2}}{2 T_{m}} \frac{\partial T_{m}}{\partial r}\right)\right|_{r=R}, \\
R(0) & =R_{0}, \quad \frac{d R(0)}{d t}=0,
\end{aligned}
$$

where $q_{h}$ is the latent heat of LOx vaporization. Additional conditions are presented in (Fig. 2).

The simulations were done using Godunov's scheme with variable time-step for stiff problem. The time step was varied depending on the maximum of the time derivatives of bubble radius, temperature and pressure. In particular we use Monotone Upstream-centered Schemes for Conservation Laws (MUSCL) based numerical scheme that extends the Godunov's scheme idea of linear piecewise approximation to each cell by using slope limited left and right extrapolated states.

List of all constants used in the simulations are represented in Table I.
1 Cole, M.D., "Challenger: America's space tragedy", Springfield, N.J., Enslow Publishers, 1995.
2 Diane, V., "The Challenger Launch Decision: Risky Culture, Technology, and Deviance at NASA", Chicago: Uni- 
TABLE I: Parameters of oxygen and hydrogen in SI units used in the simulations

\begin{tabular}{|c|c|c|c|}
\hline Parameter & Oxygen & Hydrogen & Meaning \\
\hline$c_{L}$ & 1700 & - & specific heat of the liquid \\
\hline$\rho_{L}$ & 1141 & - & liquid density \\
\hline$V_{L}$ & $1.88 \cdot 10^{-4}$ & - & $\begin{array}{l}\text { kinematic viscousity of } \\
\text { liquid }\end{array}$ \\
\hline$C_{0}$ & 1130 & - & sound velocity $(\mathrm{T}=90 \mathrm{~K})$ \\
\hline$T_{c}$ & 154.58 & - & critical temperature \\
\hline$p_{c}$ & $5.043 \cdot 10^{6}$ & - & critical pressure \\
\hline$\lambda$ & 7 & - & factor \\
\hline$q_{h}$ & $2.13 \cdot 10^{5}$ & - & specific heat of evaporation \\
\hline$C_{v}$ & 653 & 10130 & $\begin{array}{l}\text { specific heat of vapor } \\
\text { at } V=\text { const, } T=300 \mathrm{~K}\end{array}$ \\
\hline$R_{v}$ & 264 & 4124 & gas constant \\
\hline$C_{p}$ & 917 & 14270 & $\begin{array}{l}\text { specific heat of vapor } \\
\text { at } \mathrm{p}=\mathrm{const}, \mathrm{T}=300 \mathrm{~K}\end{array}$ \\
\hline$\gamma$ & 1.4 & 1.4 & \\
\hline$\kappa_{i}$ & 0.0565 & 0.0165 & $\begin{array}{l}\text { thermal conductivity of } \\
\text { saturated vapor }(T=80 \mathrm{~K})\end{array}$ \\
\hline$\kappa_{L}$ & 0.17 & - & $\begin{array}{l}\text { thermal conductivity } \\
\text { of liquid }(\mathrm{T}=80 \mathrm{~K})\end{array}$ \\
\hline$Q_{h}$ & $1.418 \cdot 10^{8}$ & - & heat of combustion \\
\hline$\sigma$ & $7.3 \cdot 10^{-3}$ & - & liquid surface tension \\
\hline$D_{i}$ & - & $6.5 \cdot 10^{-5}$ & $\begin{array}{l}\text { diffusion constant } \\
(\mathrm{T}=270 \mathrm{~K}, \mathrm{p}=1 \mathrm{~atm})\end{array}$ \\
\hline
\end{tabular}

versity Of Chicago Press, 1996.

3 Osipov, V.V., Muratov, C. B., , Hafiychuk, H,
Ponizovskaya-Devine, K, Smelyanskiy, V, Mathias, D, Lawrence, S, and Werkheiser, M, "Hazards Induced by Breach of Liquid Rocket Fuel Tanks: Risks of Cryogenic H2/Ox Fluid Explosions", (arXiv:1012.5135v1).

4 Brennen, C. E., "Fundamentals of Multiphase Flow", Cambridge University Press, 2005; Brennen, C. E., "Cavitation and Bubble Dynamics", Oxford University Press, 1995.

${ }^{5}$ Fujikawa, S. and Akamatsu, T., "Effects of the nonequilibrium condensation of vapor on the pressure wave produced by the collapse of the bubble in a liquid", J. Fluid Mech., 97, part 3, 481-512 (1980).

6 Landau,L. D. and Lifshits, E. M., "Course of Theoretical Physics: Hydrodynamics", v. 6, Pergamon Press, London, 1987.

7 Ripley D.L, Gardiner W.C. Jr, "Shock tube study of the hydrogen-oxygen reaction. II. Role of exchange initiation", J. Chem. Phys., 44, 2285 (1966); Michael, J.V., Sutherland, J.W., Harding, L.B., Wagner, A.F., "Initiation in $\mathrm{H} 2$ /O2: rate constants for $\mathrm{H} 2+\mathrm{O} 2 \rightarrow \mathrm{H}+\mathrm{HO} 2$ at high temperature", Proceedings of the Combustion Institute, 28, 1471-1478 (2000).

8 Mitani, T. and Williams, F. A., "Studies of Cellular Flames in Hydrogen-Oxygen-Nitrogen Mixtures", Combustion and Flame, 39, 169 (1980).

9 Kao, S. and Shepherd, J. E., "Numerical solution methods for control volume explosions and ZND detonation structure", GALCIT Report FM2006.007 (2008).

10 Clark, J. A., "Universal Equations for Saturation Vapor Pressure", 40th AIAA/ASME/SAE/ASEE Joint Propulsion Conference and Exhibit, Fort Lauderdale, Florida, July 2004.

11 Osipov, V. V., Muratov, C. B., "Dynamic condensation blocking in cryogenic refueling", APL, 93, 224105 (2008).

12 Lifshits, E. M. and Pitaevskii, L. P. , "Physical Kinetics", Pergamon Press, Oxford, 1981. 